\title{
Global Individual Ancestry Using Principal Components for Family Data
}

\author{
Mariza de Andrade ${ }^{a}$ Debashree Ray ${ }^{b}$ Alexandre C. Pereira ${ }^{c}$ Júlia P. Soler ${ }^{d}$ \\ ${ }^{a}$ Division of Biomedical Statistics and Informatics, Mayo Clinic, Rochester, Minn., and bepartment of Biostatistics, \\ University of Minnesota, Minneapolis, Minn., USA; ${ }^{C}$ Heart Institute, Medical School, and ${ }^{\mathrm{d}}$ Department of Statistics, \\ University of São Paulo, São Paulo, SP, Brazil
}

\section{Key Words}

Association analysis · Multivariate polygenic mixed models .

Kinship matrix $\cdot$ Admixed population

\begin{abstract}
Studies of complex human diseases and traits associated with candidate genes are potentially vulnerable to bias (confounding) due to population stratification and inbreeding, especially in admixed population. In GWAS, the principal components (PCs) method provides a global ancestry value per subject, allowing corrections for population stratification. However, these coefficients are typically estimated assuming unrelated individuals, and if family structure is present and ignored, such substructures may induce artifactual PCs. Extensions of the PCs method have been proposed by Konishi and Rao [Biometrika 1992;79:631-641], taking into account only siblings' relatedness, and by Oualkacha et al. [Stat Appl Genet Mol Biol 2012, DOI: 10.2202/15446115.1711], taking into account large pedigrees and highdimensional phenotype data. In this work, we extend these methods to estimate the global individual ancestry coefficients from PCs derived from different variance component matrix estimators using SNPs from two simulated data sets
\end{abstract}

and two real data sets: the GENOA sibship data consisting of European and African-American subjects and the Baependi Heart Study consisting of 80 extended Brazilian families, both with genotyping data from the Affymetrix 6.0 chip. Our results show that the family structure plays an important role in the estimation of the global individual ancestry value for extended pedigrees but not for sibships.

두 2015 S. Karger AG, Base

\section{Introduction}

Studies of complex human diseases and traits associated with candidate genes are potentially vulnerable to bias (confounding) due to population stratification and inbreeding, especially in admixed population. To correct for population stratification in GWAS, several approaches have been proposed such as genomic controls [1] and principal components (PCs) $[2,3]$. The advantages of using PCs are that they can be applied to discrete and continuous variables, they can correct for population stratification, and they also provide a global ancestry value per subject as implemented in the EIGENSOFT software [2]. The disadvantages of PCs are that they are only applicable

\section{KARGER 125}

(c) 2015 S. Karger AG, Base

0001-5652/15/0801-0001\$39.50/0

E-Mail karger@karger.com

www.karger.com/hhe
Mariza de Andrade

Division of Biomedical Statistics and Informatics, Mayo Clinic 2001 st Street SW

Rochester, MN 55905 (USA)

E-Mail mandrade@mayo.edu 
to uncorrelated data sets and that they cannot handle admixed populations in family data.

There are several packages available that take into account admixture, calculating not only the global ancestry value but also the local individual ancestry coefficients; but these have limitations. For instance, the program STRUCTURE can handle more than two ancestral populations, but it cannot handle large numbers of SNPs, and it can only be applied to unrelated subjects $[4,5]$. On the other hand, the program HAPMIX can handle genomewide genotype data, but it is only applicable to two ancestral populations and unrelated subjects [6]. Recently, a PC-based algorithm for determining ancestry along each chromosome from a high-density, genome-wide set of phased SNP genotypes of admixed individuals (PCAdmix) has been developed for more than 3-population admixture [7]. When the data consist of families or related subjects, one alternative has been proposed to calculate the eigenvectors (or their loadings) on the founders and married-ins only (i.e., the unrelated subjects). Then, using these eigenvectors, one can determine the PCs for the related subjects as implemented in the R package SNPRelate [8]. However, when using these programs, if family structure is present and ignored, such substructures may induce artifactual PCs.

PCs for related subjects were first proposed by Konishi and Rao [9] for families with different numbers of siblings by taking into account between-family variability; they highly recommended the use of a consistent estimator for the kinship matrix to calculate the eigenvectors in family data. Ott and Rabinowitz [10] introduced PCs of heritability $(\mathrm{PCH})$ that capture the familial information across phenotypes by calculating linear combinations of traits that maximize the heritability for combined phenotypes for family data. They showed that the first $\mathrm{PCH}$ as a quantitative trait in linkage analysis provides a gain in power compared to the standard PC analysis. Within the PCH framework, Wang et al. [11] proposed a ridge-penalized $\mathrm{PC}$ approach based on heritability $\left(\mathrm{PCH}_{\lambda}\right)$ for high-dimensional family data by adding a penalty to the subject-specific variation. Oualkacha et al. [12] proposed an ANOVA estimator for the variance components in general pedigrees and high-dimensional family data using the PCH framework. By using simulated pedigree data, they showed that their proposed method yields similar results compared to the $\mathrm{PCH}$ and $\mathrm{PCH}_{\lambda}$.

The goal of our paper is to incorporate SNPs instead of quantitative traits in the $\mathrm{PCH}_{\lambda}$ approach proposed by Oualkacha et al. [12] to calculate PCs that simultaneous- ly correct for population stratification taking into account the family structure. For admixed populations, Thornton and colleagues $[13,14]$ proposed a method that takes into account the admixture in the kinship coefficients by modeling them as a random effect using SNP data. Their method captures very well the relatedness between admixed subjects, since it uses the ancestral population's allele frequency for each SNP. The difference between these approaches is that the latter includes the admixture in the polygenic random effect, i.e., the elements of the kinship matrix are estimated taking into account the proportions of three ancestry populations (European, African, and Asians), and our approach is to correct for population stratification using the PCs taking into account the family structure in the fixed effect.

In the Material and Methods section, we describe the methods to calculate PCs and the data sets used: one simulated data and two real data (the GENOA sibship data consisting of European and African-American subjects $[15,16]$ and the Baependi Heart Study consisting of 80 extended families collected from the highly admixed Brazilian $[17,18]$ population, both with SNP data from the Affymetrix 6.0 chip). Then, we show our results using the simulated and real data sets and the approaches described previously. In the last two sections, we summarize our findings, point out their advantages and limitations, and conclude with final observations. In addition, online supplementary materials are also provided for a further clarification of the proposed methods.

\section{Material and Methods}

\section{PC Analysis for Extended Pedigrees}

Let $\mathbf{Y}_{f}=\left(\mathbf{y}_{f 1}^{\prime}, \ldots, \mathbf{y}_{f p}^{\prime}\right)$ be a $\left(n_{f} p \times 1\right)$ vector for all $p$ variables and all members of the $f$-th family, with $E\left(\mathbf{Y}_{f}\right)=1_{f} \otimes \mu_{f}$ and $\operatorname{Cov}\left(\mathbf{Y}_{f}\right)=$ $2 \Phi_{f} \otimes \Sigma_{g}+I_{f} \otimes \Sigma_{e}$, where $\mu=\left(\mu_{1}^{\prime}, \ldots, \mu_{p}^{\prime}\right)^{\prime}$ is the $(p \times 1)$ mean vector for all $p_{f}$ variables, with $\Sigma_{g}$ and $\Sigma_{e}$ as the $(p \times p)$ covariance matrix for $p$ variables associated with polygenic and error component, respectively. For all $F$ families, we define $\mathbf{Y}=\left(\mathbf{Y}_{1}^{\prime}, \ldots, \mathbf{Y}_{\mathrm{F}}^{\prime}\right)$ as a $(N p$ $\times 1)$ vector containing all $p$ variables for all individuals, with $N=$ $\sum_{i=1}^{F} n_{f}$ with $E(\mathbf{Y})=\mathbf{1}_{N} \otimes \mu_{f}$ and $\operatorname{Cov}(\mathbf{Y})=\operatorname{Diag}\left(2 \Phi_{f}\right) \otimes \Sigma_{g}+I_{N} \otimes$ $\Sigma_{e}, f=1, \ldots, F$. This framework represents the multivariate familybased model $[10,12,19,20]$. In this paper, the $p$ variables represent the SNP genotypes (standardized or not) selected from the whole genome to estimate the global ancestry coefficients for individuals in family structures. More details about the model described here and the approaches described below are available in de Andrade and Soler [21].

\section{PC Analysis for Family Data}

Using the model described above, the PCs are calculated using unbiased or consistent estimators of the covariance matrices $\Sigma_{g}$ and $\Sigma_{e}$. Konishi and Rao [9] proposed ANOVA-based estimators 
for these covariance matrices using nuclear families. From now on, the superscript $K$ will represent this method [9]. For sibships of any size, the kinship matrix is given by $2 \Phi_{f}=1_{n_{\mathrm{f}}} 1_{n_{\mathrm{p}}}$, and by using the model described above, the estimators for the covariance matrices can be obtained from the classical ANOVA results, as shown below,

$$
\begin{aligned}
& \hat{\sum}_{e}^{K}=\frac{\mathbf{S}_{w}}{N-F}, \\
& \hat{\sum}_{g}^{K}=N_{0}^{-1}\left(\frac{\mathbf{S}_{b}}{F-1}-\frac{\mathbf{S}_{w}}{N-F}\right),
\end{aligned}
$$

with

$$
N_{0}=\frac{N-\left(\Sigma_{f} n_{f}^{2} / N\right)}{F-1},
$$

and $\mathbf{S}_{w}$ and $\mathbf{S}_{b}$ as the sum of square and cross-product matrices within and between families, respectively. Thus, the PCs can be obtained using the spectral decomposition of the matrices $\hat{\Sigma}^{K}, \hat{\Sigma}_{g}^{K}$, and $\hat{\Sigma}_{e}^{K}$ such as with $\Sigma^{K}=\Sigma_{w}^{K}+\Sigma_{b}^{K}$.

For the standardized data, we use $\hat{\mathbf{R}}_{g}^{K}=\mathbf{D}^{K} \hat{\mathbf{\Sigma}}_{g}^{K} \mathbf{D}^{K}$ and $\hat{\mathbf{R}}_{e}^{K}=$ $\mathbf{D}^{K} \hat{\Sigma}_{e}^{K} \mathbf{D}^{K}$, with $\mathbf{D}^{K}$ as the diagonal matrix with elements $\left(s_{j j}^{K}\right)^{-1 / 2}$, and $s_{j j}^{K}$ as the diagonal elements of $\hat{\Sigma}^{K}, j=1,2, \ldots, p$.

When the multivariate family-based model described above is extended for general pedigrees, Oualkacha et al. [12] proposed to use ANOVA estimators for the variance component matrices. By using the sum of square and cross-product matrices $\mathbf{S}_{w}$ and $\mathbf{S}_{b}$ in equations 1 and 2, the estimators of the covariance matrices are written as

$$
\begin{aligned}
& \hat{\Sigma}_{g}^{A}=\frac{\mathbf{S}_{b} /(F-1)-\mathbf{S}_{w} /(N-F)}{\left(\sigma_{c}-\frac{\sigma_{b}}{N}\right) /(F-1)-\left(\sigma_{a}-\sigma_{c}\right) /(N-F)}, \\
& \hat{\sum}_{e}^{A}=\frac{1}{N-F} \mathbf{S}_{w}-\frac{\left(\sigma_{a}-\sigma_{c}\right)}{N-F} \hat{\Sigma}_{g}^{A},
\end{aligned}
$$

where

$$
\begin{aligned}
& \sigma_{a}=\sum_{f=1}^{F} \sigma_{a}^{(f)}, \sigma_{b}=\sum_{f=1}^{F} \sigma_{b}^{(f)}, \sigma_{c}=\sum_{f=1}^{F} \frac{1}{n_{f}} \sigma_{b}^{(f)}, \sigma_{a}^{(f)}=\operatorname{tr}\left(2 \Phi^{(f)}\right), \\
& \text { and } \sigma_{b}^{(f)}=\sum_{j=1}^{n f} \sum_{k=1}^{n f} \Phi_{j k}^{(f)} .
\end{aligned}
$$

From now on, the superscript $A$ will represent the Oualkacha method. When $2 \Phi_{f}=1_{n_{\mathrm{f}}} 1_{n_{\mathrm{p}}}$ the estimators in equations 2 and 3 are the same ANOVA estimators as in equations 1 and 2, as proposed by Konishi and Rao [9]. The PCs are obtained by the spectral decomposition of the matrices $\hat{\boldsymbol{\Sigma}}^{A}, \hat{\boldsymbol{\Sigma}}_{g}^{A}$, and $\boldsymbol{\Sigma}_{e}^{A}$ with $\hat{\boldsymbol{\Sigma}}^{A}=\hat{\boldsymbol{\Sigma}}_{g}^{A}+\hat{\boldsymbol{\Sigma}}_{e}^{A}$. Furthermore, we can also use the correspondent correlation matrices, proposed by Bilodeau and Duchesne [22], for equations 2 and 3 to calculate the PCs, given by $\hat{\mathbf{R}}_{g}^{A}=\mathbf{D}^{A} \hat{\boldsymbol{\Sigma}}_{g}^{A} \mathbf{D}^{A}$ and $\hat{\mathbf{R}}_{e}^{A}=\mathbf{D}^{A} \hat{\boldsymbol{\Sigma}}_{e}^{A} \mathbf{D}^{A}$, where $\mathbf{D}^{A}$ is a diagonal matrix with elements $\left(s_{j j}^{A}\right)^{1 / 2}$, and $s_{j j}^{A}$ as the diagonal elements of $\hat{\Sigma}^{A}, j=1,2, \ldots, p$.

We also apply the PCH proposed by Ott and Rabinowitz [10] and extended by Oualkacha et al. [12] to determine the PCs for extended pedigrees, i.e., instead of looking for the linear combinations of phenotypes with maximum variance, we focus on the linear combinations of phenotypes that maximize their heritability taking into account their within-family correlation. Our goal is to find combinations of SNPs that maximize the trace of the heritabil- ity matrix $\mathbf{H}_{g}^{2}=\boldsymbol{\Sigma}_{g} /\left(\boldsymbol{\Sigma}_{g}+\boldsymbol{\Sigma}_{e}\right)^{-1}$, which is equivalent to obtain the eigenvectors $\mathbf{b}$, such that

$$
\max _{b} \frac{\mathbf{b}^{\prime} \hat{\sum}_{g}^{A} \mathbf{b}}{\mathbf{b}^{\prime} \hat{\sum}_{e}^{A} \mathbf{b}}
$$

and $\mathbf{b} \hat{\boldsymbol{\Sigma}}_{e}^{A} \mathbf{b}=1$. Then, we obtain the PCs using the eigenvectors of the generalized eigen system [23]. Due to the high-dimensional and sparse matrices $(p \gg N)$, Wang et al. [11] proposed a ridgepenalized PC approach to obtain PCH in order to accommodate large numbers of phenotypes. With this approach, one can either use the PCs from a multivariate familial covariance matrix (for unstandardized data) or those from a correlation matrix (for standardized data) as proposed by Bilodeau and Duchesne [22]. Then, the leading PC is defined as

$$
P C H_{\lambda}=\arg \max _{\|\beta\|=1} \frac{\mathbf{b}^{\prime} \sum_{\mathrm{g}}^{\mathrm{A}} \mathbf{b}}{\mathbf{b}^{\prime} \sum_{e}^{A} \mathbf{b}+\lambda\|\beta\|^{2}},
$$

with $\lambda$ as the regularization parameter to be specified. When $\lambda=0$, $\mathrm{PCH}_{\lambda}$ is the original non-penalized leading PC (PCH). When $\lambda \rightarrow$ $\infty$, the second term of the denominator of $\mathrm{PCH}_{\lambda}$ dominates, and $\mathrm{PCH}_{\lambda}$ approaches the linear combination that maximizes the between-family variation $\Sigma_{g}^{A}$, i.e., $P C B=\arg \max _{\|\beta\|=1} \mathbf{b}^{\prime} \boldsymbol{\Sigma}_{g}^{A} \mathbf{b}$. When $\lambda$ is between 0 and $\infty$, the solution corresponds to the direction that provides the optimal balance between maximizing the betweenfamily variation (PCB) and minimizing the within-family variation (PCW), defined as $P C W=\arg \max _{\|\beta\|=1} \mathbf{b}^{\prime} \boldsymbol{\Sigma}_{e}^{A} \mathbf{b}$. These PCBs are the ones we use and recommend in our association analyses.

For comparison, we assume all subjects are unrelated. Thus, the covariance matrix using unrelated subjects $\Sigma^{U}$ is estimated as

$$
\hat{\Sigma}^{U}=\frac{1}{N-1} \mathbf{S}
$$

with $\mathbf{S}=\sum_{f} \Sigma_{i}\left(y_{i f}-\bar{y}\right)\left(y_{i f}-\bar{y}\right)^{\prime}=\mathbf{S}_{w}+\mathbf{S}_{b}$, and $\bar{y}=\hat{\mu}=1 / N \mathbf{Y}_{N \times p}^{\prime} 1_{N}$ as the overall mean with superscript $U$ representing the unrelated subjects. This approach is equivalent to the PCs estimated by Price et al. [2]. The global ancestry coefficients are obtained from the spectral decomposition of the correlation matrix, with $\hat{\mathbf{R}}^{U}=$ $\mathbf{D}^{U} \hat{\boldsymbol{\Sigma}}^{U} \mathbf{D}^{U}$, with $\mathbf{D}^{U}$ as the diagonal matrix with elements

$$
\sqrt{s_{i j}}=\sqrt{p_{j}\left(1-p_{j}\right)} \text { with } p_{j}=\frac{1+N \bar{y}_{j}}{2+2 N},
$$

$j=1,2, \ldots, p$. The global ancestry coefficients may also be obtained from the singular value decomposition of the standardized data matrix, with the $i$-th column (for the $i$-th individual) as $\mathbf{X}_{i}^{U}=$ $\left(\mathbf{Y}_{i}-\bar{Y}_{i}\right) \mathbf{D}^{U}$.

\section{Data Description}

\section{Real Data Sets}

The first data set is the GENOA sibship data that consist of European ancestry (EA: Rochester, Minn., USA) and African-American (AA: Jackson, Miss., USA) subjects with SNP data from the Affymetrix 6.0 chip $[15,16]$. Sibships with one sibling were excluded from the analysis. For the EA and AA subjects, the screened data contain 534 and 548 families with 1,386 and 1,263 individuals and data on 83,568 and 50,510 SNPs, respectively. The second data set is the Baependi Heart Study that consists of 119 extended Brazilian families with 1,712 individuals and SNP data from the Affymetrix 6.0 SNP chip [17]. Families with only one individual or unrelated individuals with genotype data were excluded due to lack of information. 
Table 1. Intercontinental autosomal genetic distances based on SNPs (pairwise $F_{S T}$ )

\begin{tabular}{llll}
\hline & CEU & Yoruba & Asian \\
\hline CEU & 0.153 & & \\
Yoruba & 0.111 & 0.190 & \\
Asian & 0.110 & 0.192 & 0.007 \\
\hline
\end{tabular}

Table 2. Distribution of the family sizes from the GENOA study by EA and AA participants

\begin{tabular}{rrr}
\hline$N_{f}$ & EA individuals & AA individuals \\
\hline 2 & 264 & 101 \\
3 & 101 & 92 \\
4 & 37 & 38 \\
5 & 24 & 15 \\
6 & 9 & 17 \\
7 & 6 & 4 \\
8 & 4 & 4 \\
9 & 3 & 3 \\
10 & 1 & - \\
11 & 1 & - \\
12 & 1 & - \\
14 & - & 1 \\
17 & 1 & - \\
\hline
\end{tabular}

$N_{f}$ represents the number of siblings per family.

Simulated Data Sets

The simulated data consist of 100 families with 20 subjects. Each family is assumed to have the same pedigree structure with 4 generations and 6 founders as described in figure 1 . The data were simulated under four scenarios, 100 SNPs and 3,000 SNPs, and with $F_{S T}=0.05$ and 0.20 . The genotype data were simulated using the Balding-Nichols model with allele frequencies for two distinct ancestral populations [24]. The allele frequency for the ancestral population $p_{a p}$ for all SNPs was generated independently from a uniform distribution: $U(0.1,0.9)$. The allele frequencies, in the subpopulations separated by Wright's $F_{S T}$, were randomly selected from a beta distribution,

$$
\beta\left(\left(\frac{1-F s t}{F s t}\right) p_{a p},\left(\frac{1-F s t}{F s t}\right)\left(1-p_{a p}\right)\right)
$$

(table 1). The admixture proportions for the 6 founders were randomly selected from $\mathrm{U}(0,1)$. Genotype data at each SNP for the 6 founders were generated based on the individual's admixture proportions for the two subpopulations, and those for the 14 non-founders were generated by dropping alleles down the pedigree. We used the R program pca_admixture_unrelateds_and_ pedigrees.r to simulate the data.

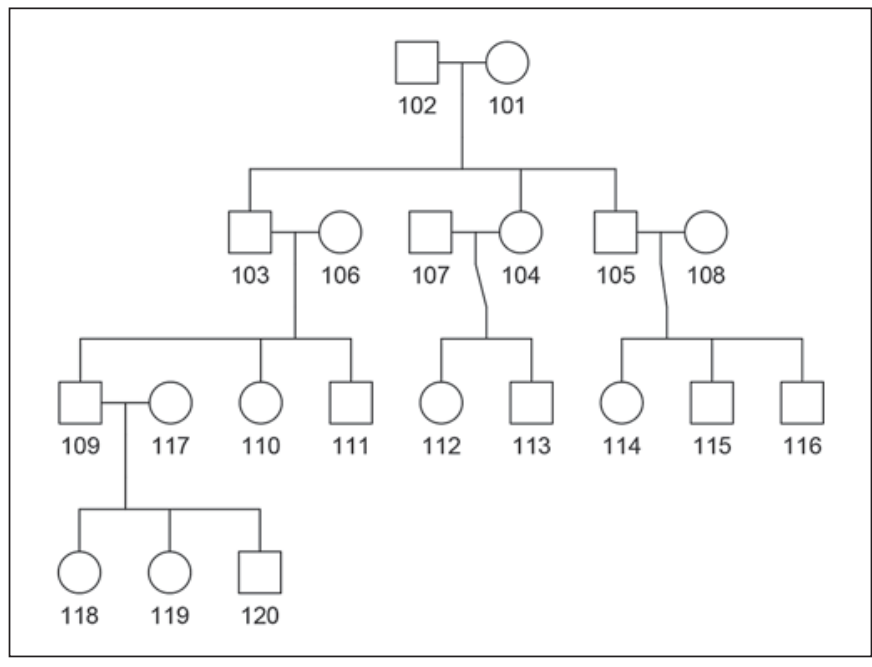

Fig. 1. The pedigree structure used for each family in the simulated data set. Squares $=$ males; circles $=$ females.

\section{Results}

For the sibship GENOA data set, we used the following exclusion criteria for each data set using PLINK [25]: subjects with $>5 \%$ missing genotype information were screened out, SNPs with a MAF $\leq 5 \%$, missingness $\leq 5 \%$, LD $(\mathrm{VIF}=1.11$ with a window size of 50 and a window shift of 5 SNPs) after linkage disequilibrium screening (- thin 0.2 ), a HWE p value $\leq 0.05$, plus SNPs in the HLA region, chromosomes 8 and 17 inversion regions, $\mathrm{X}, \mathrm{Y}$, and MT. The two final data sets included 9,224 common SNPs and 2,383 individuals (EA: 1,304; AA: 1,079) from 816 sibships (EA: 452; AA: 364 ). A detailed description of the sibship sizes and the number of families is given in table 2.

For this sibship data, the PCs using Konishi and Rao's [9] approach performed similarly for standardized and unstandardized genotype data (fig. 2a, b). Furthermore, the decomposition of the matrix $\hat{\Sigma}_{g}^{K}$ was more powerful and robust compared to the decomposition of the residual covariance matrix $\hat{\Sigma}_{e}^{K}$ and provided similar results for the total covariance matrix $\hat{\Sigma}_{t}^{K}$ (data not shown). This is due to the fact that the error covariance matrix $\hat{\Sigma}_{e}^{K}$ is close to a null matrix, i.e., $\hat{\Sigma}_{g}^{K} \cong \hat{\Sigma}^{K}$. However, when we assume that the data are unrelated, the PCs using Price et al.'s [2] approach show more variability within subjects and are sensitive to standardization, i.e., the unstandardized PC results have the power to discriminate the two racial groups, but they are not sensitive to detect outlier families (fig. 2c, d). Despite the fact that the PC plots are similar between the two approaches, the proportion of variance 


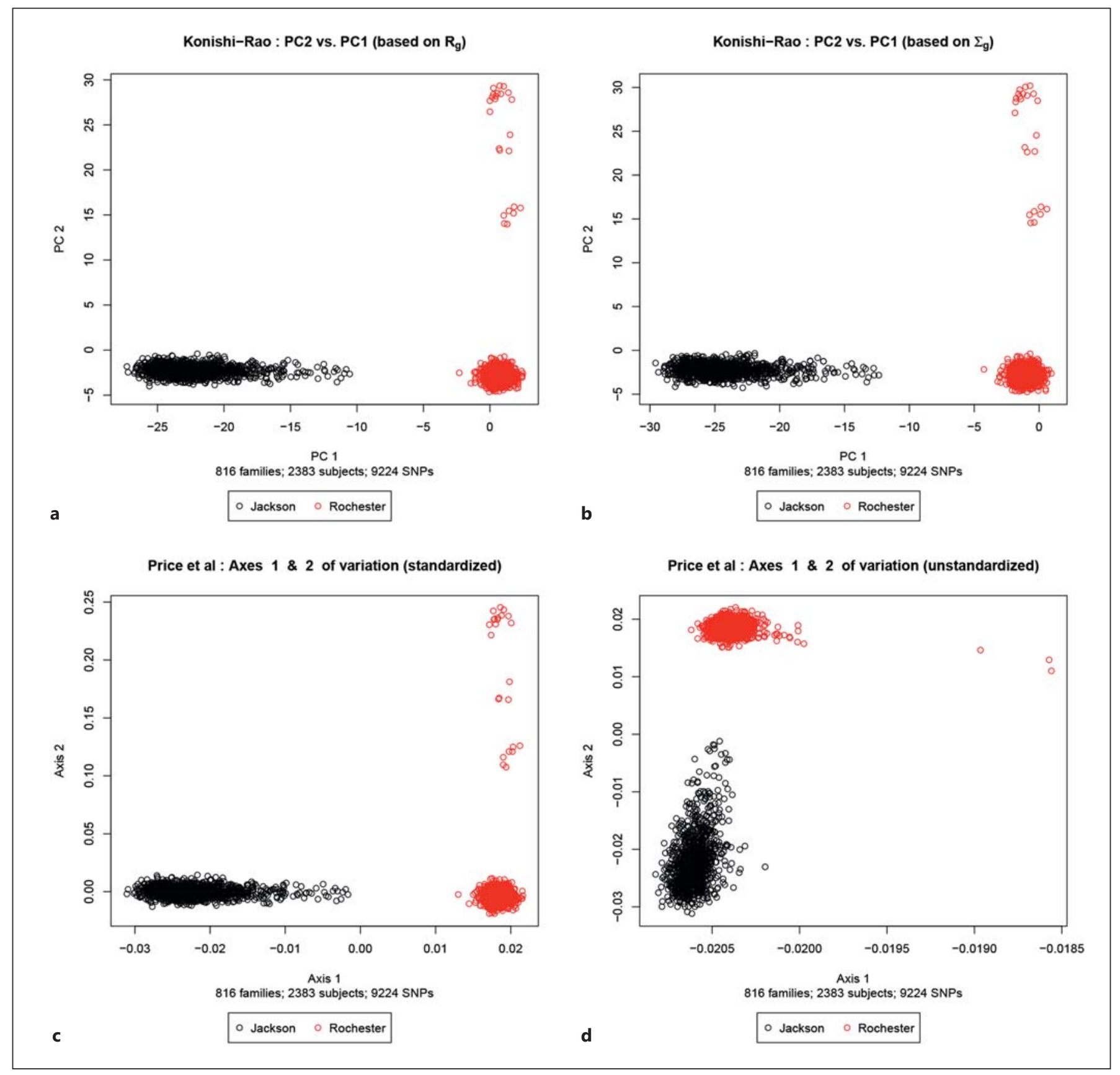

Fig. 2. a, b PCs for standardized $\Sigma_{g}^{K}$ and unstandardized $\Sigma_{g}^{K}$. c, d Variability and sensitivity of Price et al.'s [2] PCs using the GENOA sibship data.

explained by their PCs differs. For the standardized data, the sum of the three first PCs obtained by the KonishiRao method was $10.1 \%$ compared to $4.98 \%$ obtained by the method by Price et al. (table 3; online suppl. fig. S1; see www.karger.com/doi/10.1159/000381908 for all online suppl. material).

Global Individual Ancestry Using PCs for Family Data
For the Baependi data set, the same exclusion criteria were used as for the GENOA data. The final data set includes 80 families (1,109 individuals) and 8,764 SNPs. A detailed description of the sibship sizes and numbers of families is given in table 4. For this family data, both Oualkacha et al.'s [12] and Price et al.'s [2] standardized PCs 
Table 3. Proportion of variation explained by the first 10 PCs from Price et al.'s [2] and Konishi and Rao's [9] methods for standardized values using the GENOA data

\begin{tabular}{rll}
\hline PCs & Price et al. & Konishi and Rao \\
\hline 1 & 0.044 & 0.089 \\
2 & 0.0033 & 0.0069 \\
3 & 0.0025 & 0.0052 \\
4 & 0.0024 & 0.0048 \\
5 & 0.0023 & 0.0046 \\
6 & 0.0021 & 0.0044 \\
7 & 0.0020 & 0.0042 \\
8 & 0.0020 & 0.0040 \\
9 & 0.0019 & 0.0039 \\
10 & 0.0019 & 0.0039 \\
\hline
\end{tabular}

Table 4. Distribution of the family size for the Baependi Heart Study

\begin{tabular}{rlll}
\hline$N_{f}$ & Frequency, $\mathrm{n}$ & $N_{f}$ & Frequency, $\mathrm{n}$ \\
\hline 2 & 2 & 16 & 4 \\
3 & 8 & 18 & 3 \\
4 & 6 & 19 & 1 \\
5 & 8 & 21 & 3 \\
6 & 5 & 24 & 1 \\
7 & 1 & 27 & 1 \\
8 & 4 & 32 & 1 \\
9 & 6 & 46 & 1 \\
10 & 5 & 48 & 1 \\
11 & 6 & 60 & 1 \\
12 & 1 & 61 & 1 \\
13 & 5 & 68 & 1 \\
14 & 3 & 93 & 1 \\
\hline
\end{tabular}

$N_{f}$ represents the number of subjects per family.

show a similar population structure pattern with two long arms connected (fig. 3a, b). Using PCs 2 and 3, Oualkacha et al.'s results show much more population structure than Price et al.'s results, indicating that the first three PCs from Oualkacha et al. are more informative than those from Price et al. (fig. 3c, d). Furthermore, Oualkacha et al.'s results allow more dispersion among individuals than Price et al.'s results, mainly for individuals allocated between the long arms, indicating the relevance of the family relatedness (fig. 4) and that the population stratification information is retrieved from $\Sigma_{g}$ (the kinship matrix) and not from $\Sigma_{e}$. This can be further observed in table 5 and online supplementary figure S2, which show that the proportion of variation explained by the first three PCs from Oualkacha et al. was $19.1 \%$ compared to 4.4\% from Price et al.'s first three PCs.

Since we observed this difference in the proportion of variance between these two approaches using the admixed family data from Baependi, we also compared the kinship values derived from the IBD method implemented in PLINK [25] and from the REAP approach that used the allele frequencies from the three ancestral populations [14]. We observed that the relatedness coefficients obtained from these two programs were similar (online suppl. fig. S3). Furthermore, when we compared the association results for the quantitative trait average systolic blood pressure, using the $\mathrm{R}$ function in the $\mathrm{R}$ library, with the kinship matrix implemented in the R library and in the REAP program, including only age and sex as covariates (left panel of online suppl. fig. S4), and also adding PCB1 and PCB2 as covariates (right panel of online suppl. fig. S4), the results were almost identical, indicating that similar results were found by correcting the possible confounding due to population stratification through fixed or random effect. In addition, by not including the ancestral populations to estimate the kinship values, the association results in admixed families were not affected.

For the simulated data, we ran two different sets of simulation, with 100 and 3,000 SNPs, with the same family structure and size, i.e., 20 members. For the results presented here we used $F_{S T}=0.2$ only; the results are similar when using $F_{S T}=0.05$ (data not shown). Figure 5a, b show PCs 1 and 2 from Price et al.'s and Oualkacha et al.'s approaches for 100 SNPs. We did not observe any difference between the plots except for the different scales of the axes; however, we found the same difference pattern in the proportion of variance due to the PCs as in the real data sets (table 6; online suppl. fig. S5), i.e., the proportion explained by Oualkacha et al.'s approach for the first three PCs was $17.9 \%$ compared to $9.14 \%$ explained by Price et al.'s first three PCs. When we increased the number of SNPs to 3,000, we observed that PCs 1 and 2 from Oualkacha et al.'s approach could better capture the population substructure by taking into account the family structure (fig. 5c, d). For a better visualization, we selected four families at random and highlighted them in figure 5. One can observe the similar results from the Baependi data (fig. 4), i.e., the families were well separated using Oualkacha et al.'s approach. The admixture proportions for each member of these four simulated families are shown in online supplementary figure S6.

All analyses were performed using the $\mathrm{R}$ 3.0.2 package [26]. The functions that calculated the familial PC are available upon request from the authors. 


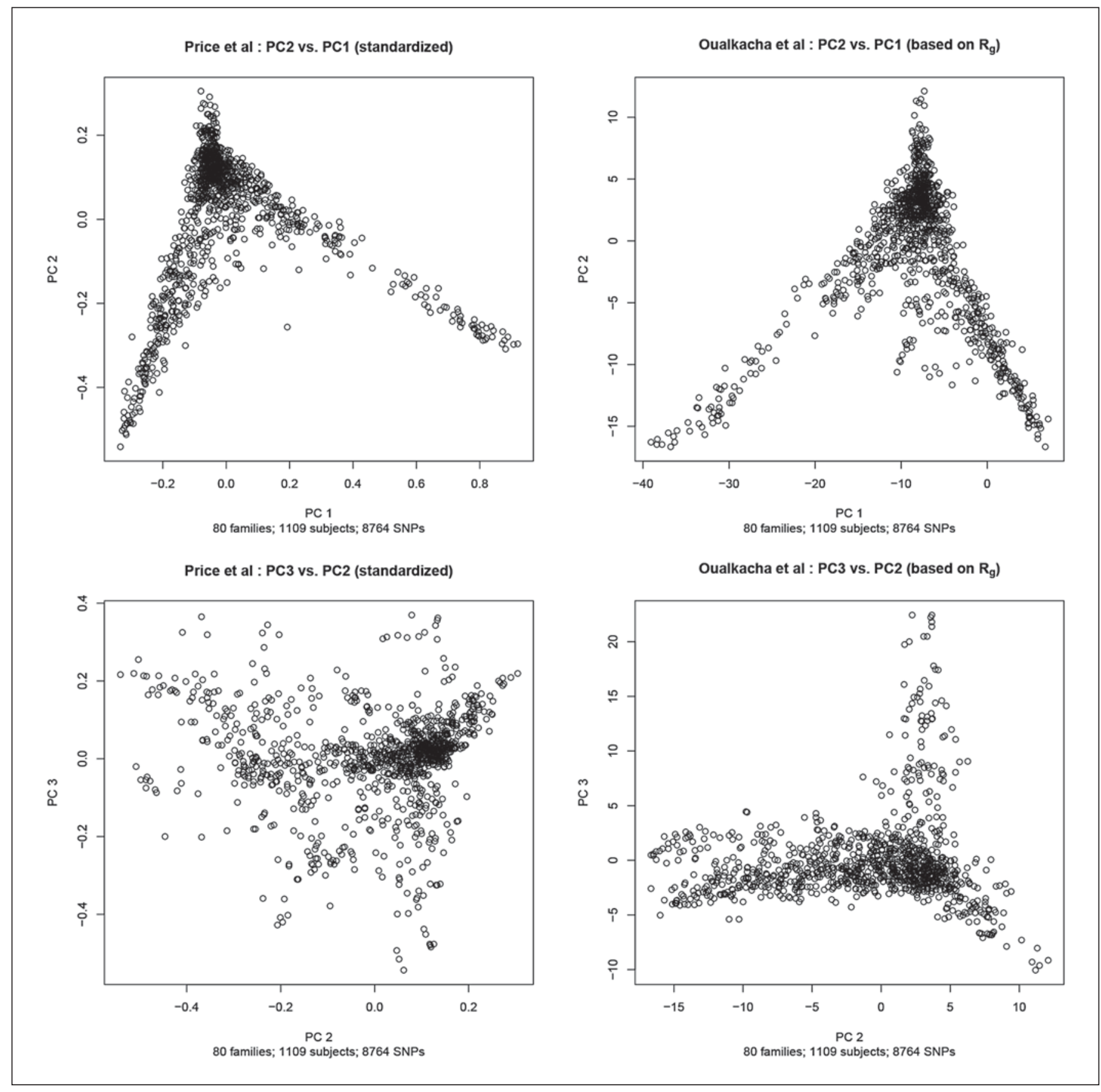

Fig. 3. Comparison of Price et al.'s [2] and Oualkacha et al.'s [12] PCs using the Baependi family data. a, b The plots of PC 1 and PC 2 are very similar between the two methods despite the scale difference. c, $\mathbf{d}$ For the plots of PC 2 and PC 3, the two methods are drastically different, however.

\section{Discussion}

It is the common practice in genome-wide association analysis to adjust for PCs to correct for population stratification; however, for cluster-correlated data such as fam- ilies, the family structure is usually ignored, and the PCs are estimated by using the loadings of the founders [8] or assuming that all subjects are unrelated [2]. This may work for a homogeneous population, but it was unclear if the same applied to admixed population. Admixture can 


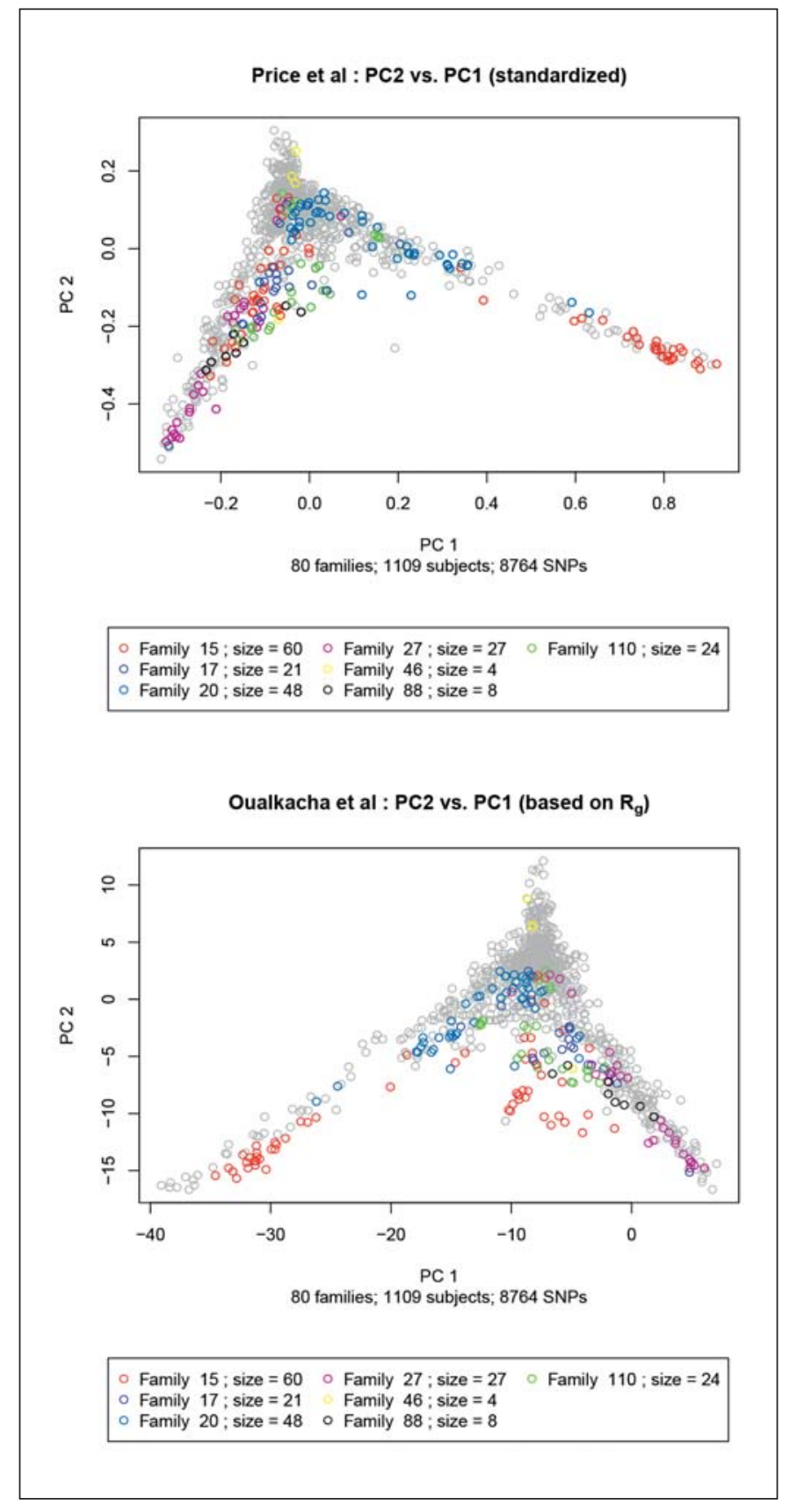

Fig. 4. Distribution of some families, selected by size, from the Baependi data in Price et al.'s [2] and Oualkacha et al.'s [12] PC plots. One can observe that the arms of the PCs differ between the two methods.

be used as a tool for finding linked genes and also to detect differences from allelic association between loci [27]. It is also well known that the effect of population stratification in unrelated subjects in association analysis can induce false-positive results due to linkage disequilibrium and
Table 5. Proportion of variation explained by the first 10 PCs from Price et al.'s [2] and Oualkacha et al.'s [12] methods for standardized values using the Baependi data

\begin{tabular}{rll}
\hline PCs & Price et al. & Oualkacha et al. \\
\hline 1 & 0.022 & 0.086 \\
2 & 0.014 & 0.070 \\
3 & 0.008 & 0.035 \\
4 & 0.0069 & 0.031 \\
5 & 0.0068 & 0.028 \\
6 & 0.0061 & 0.026 \\
7 & 0.0059 & 0.0255 \\
8 & 0.0055 & 0.0249 \\
9 & 0.0053 & 0.0246 \\
10 & 0.005 & 0.0239 \\
\hline
\end{tabular}

Table 6. Proportion of variation explained by the first 10 PCs from Price et al.'s [2] and Oualkacha et al.'s [12] methods (standardized versions) for 100 families (2,000 subjects) and 100 and 3,000 SNPs

\begin{tabular}{|c|c|c|c|c|}
\hline \multirow[t]{2}{*}{ PC } & \multicolumn{2}{|l|}{100 SNPs } & \multicolumn{2}{|l|}{ 3,000 SNPs } \\
\hline & Price et al. & Oualkacha et al. & Price et al. & Oualkacha et al. \\
\hline 1 & 0.058 & 0.101 & 0.039 & 0.072 \\
\hline 2 & 0.017 & 0.040 & 0.004 & 0.016 \\
\hline 3 & 0.0164 & 0.038 & 0.004 & 0.016 \\
\hline 4 & 0.0163 & 0.035 & 0.004 & 0.015 \\
\hline 5 & 0.0159 & 0.033 & 0.004 & 0.015 \\
\hline 6 & 0.0157 & 0.032 & 0.0035 & 0.015 \\
\hline 7 & 0.0156 & 0.0305 & 0.0035 & 0.015 \\
\hline 8 & 0.0151 & 0.029 & 0.0035 & 0.015 \\
\hline 9 & 0.0147 & 0.028 & 0.0035 & 0.0148 \\
\hline 10 & 0.0145 & 0.014 & 0.0034 & 0.0147 \\
\hline
\end{tabular}

allele frequency distributions from different populations in candidate genes [28-30], a reasonable large number of SNPs [31], and lastly for thousands of SNPs [2]. However, for clustered data or family data, it has never been investigated whether the family structure should be taken into account when calculating the PCs for an admixed population.

Several approaches have been used to include the family structure in the estimation of the PCs in family data. The approach developed by Konishi and Rao [9] is restricted to nuclear families or sibships. By using standardized genotype data and sibships from GENOA [16], we showed that the PCs, taking or not taking the family structure into account, were similar except for the scaling difference; however, the proportion of variance ex- 


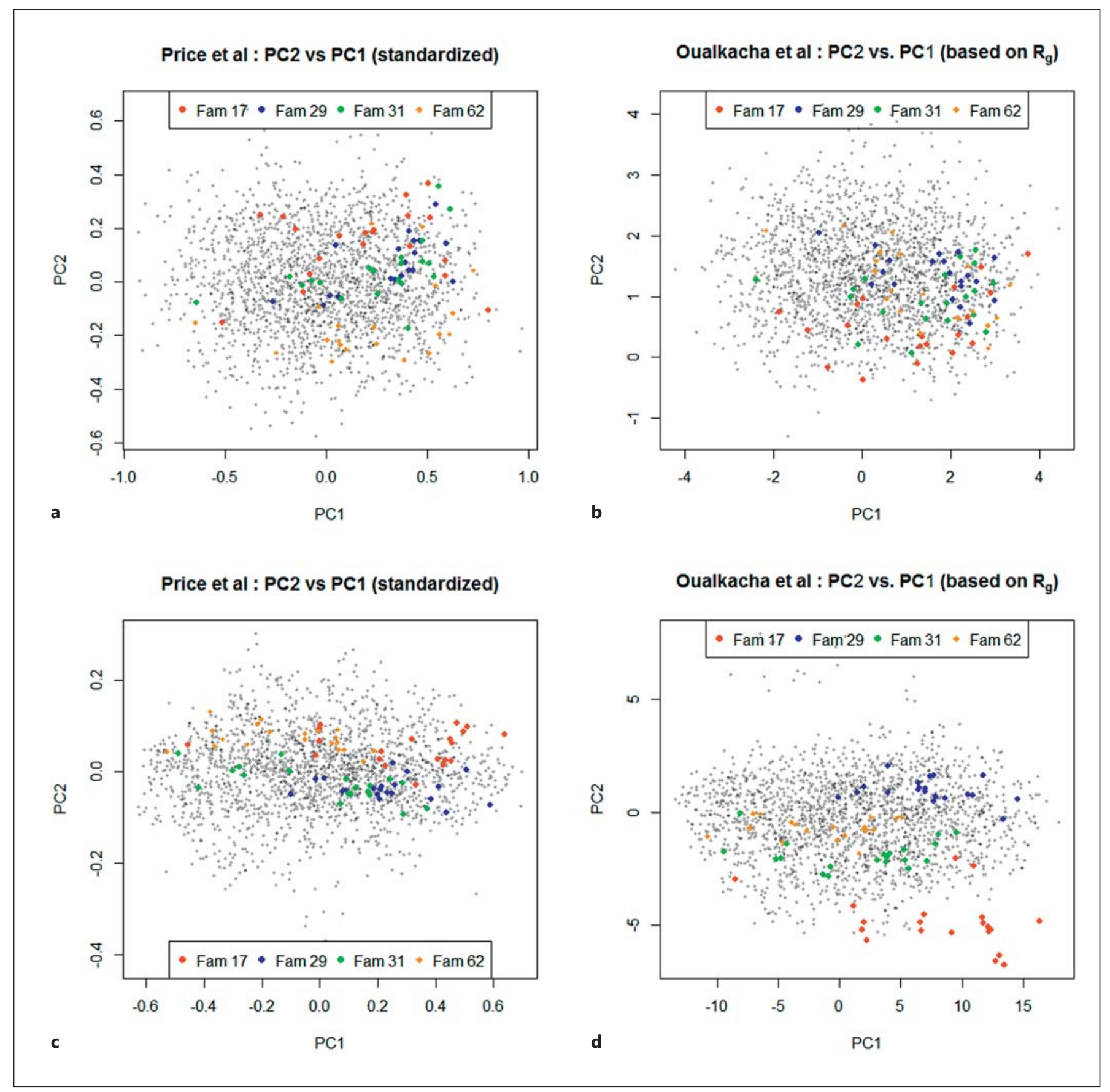

Fig. 5. Distribution of some randomly chosen families in the PC plot from Price et al.'s [2] and Oualkacha et al.'s [12] methods using standardized genotype data and simulated data from 2,000 subjects and 100 SNPs (a, b) or 3,000 SNPs (c, d).

plained by the first three PCs including the family structure is much larger than that by the PCs ignoring the family structure. With the approach proposed by us for admixed families with more than two generations, using genotype data from the Baependi study [17] and simu-

Global Individual Ancestry Using PCs for Family Data lated data, one notices that the plots based on PCs that take into account the family structure were more informative for the admixture than the plots based on PCs ignoring the family structure. Furthermore, the proportion of variance explained by the first three PCs, when 
the family structure was incorporated in the estimation of the PCs, was larger than when family structure was ignored. To investigate the effect of Price et al.'s and Oualkacha et al.'s PCs on the genome-wide association results, we performed three analyses: two including each of the two PC approaches (Price et al. and Oualkacha et al.) and one without PCs. We used the five phenotypes related to metabolic syndrome adjusted for age, age $^{2}$, and sex. We observed that when no PCs were included there was either a decrease or an increase in the significance level of SNPs depending on the phenotype; on the other hand, when the PCs were included, Oualkacha et al.'s PCs yield less significant results than Price et al.'s PCs (data not shown).

We also performed genome-wide association analyses using the Baependi data only and observed that, by not including the ancestral populations in the estimation of the kinship values, the association results were not affected (online suppl. fig. S4). Furthermore, the inclusion of the PCs in the association analysis using either of the two kinship values reduced the level of significance drastically (online suppl. fig. S4).

\section{Conclusion}

The objective of this paper was to show that the family structure should be incorporated in the estimation of the PCs in admixed populations. The conceived idea that, when analyzing family data, it is not necessary to take the family structure into account does not hold in general. This is the case for extended families from admixed populations. We have used real and simulated data sets, and both have shown the relevance of the family structure in the estimation of the PCs and their role in the association analysis.

\section{Acknowledgments}

We would like to thank DeLaine Anderson for her technical assistance with the manuscript and Dr. Thimothy Thornton for sharing his R function to simulated admixed families. The project was partially supported by the NIH grant HL87660 (to M.d.A.) and a Biomedical Statistics and Informatics (BSI) Merit Award, Department of Health Sciences Research, Mayo Clinic (to D.R.).

\section{References}

1 Devlin B, Roeder K: Genomic control for association studies. Biometrics 1999;55:9971004.

-2 Price AL, Patterson NJ, Plenge RM, Weinblatt ME, Shadick NA, Reich D: Principal component analysis corrects for stratification in genome-wide association studies. Nat Genet 2006;38:904-909.

$>3$ Patterson N, Price AL, Reich D: Population structure and eigenanalysis. PLoS Genet 2006; 2:e190.

4 Prichard JK, Stephens M, Donnelly P: Inference of population structure using multilocus genotype data. Genetics 2000;155:945-959.

5 Falush D, Stephens M, Pritchard JK: Inference of population structure using multilocus genotype data: linked loci and correlated allele frequencies. Genetics 2003;164:15671587.

6 Price AL, Tandon A, Patterson N, Barnes KC, Rafaels N, Ruczinski I, Beaty TH, Mathias R, Reich D, Myers S: Sensitive detection of chromosomal segments of distinct ancestry in admixed populations. PLoS Genet 2009;5: e1000519.

7 Brisbin A, Bryc K, Byrnes J, Zakharia F, Omberg L, Degenhardt J, Reynolds A, Ostrer H, Mezey JG, Bustamante CD: PCAdmix: principal components-based assignment of ances- try along each chromosome in individuals with admixed ancestry from two or more populations. Hum Biol 2012;84:343-364.

${ }_{8}$ Zheng X, Levine D, Shen J, Gogarten SM, Laurie C, Weir BS: A high-performance computing toolset for relatedness and principal component analysis of SNP data. Bioinformatics 2012;28:3326-3328.

$>9$ Konishi S, Rao CR: Principal components for multivariate familial data. Biometrika 1992; 79:631-641.

10 Ott J, Rabinowitz D: A principal-components approach based on heritability for combining phenotype information. Hum Hered 1999;49: 106-111.

11 Wang Y, Fang Y, Jin M: A ridge penalized principal-components approach based on heritability for high-dimensional data. Hum Hered 2007;64:182-191.

12 Oualkacha K, Labbe A, Ciampi A, Roy MA, Maziade M: Principal components of heritability for high dimension quantitative traits and general pedigrees. Stat Appl Genet Mol Biol 2012, DOI: 10.2202/1544-6115.1711.

13 Thornton T, McPeek MS: ROADTRIPS: casecontrol association testing with partially or completely unknown population and pedigree structure. Am J Hum Genet 2010;86: 172-184.
14 Thornton T, Tang H, Hoffmann TJ, OchsBalcom HM, Caan BJ, Risch N: Estimating kinship in admixed populations. Am J Hum Genet 2012;91:122-138.

15 FBPP investigators: Multi-center genetic study of hypertension: the Family Blood Pressure Program (FBPP). Hypertension 2002;39: 3-9.

16 Daniels PR, Kardia SL, Hanis CI, Brown CA, Hutchinson R, Boerwinkle E, Turner ST: Familial aggregation of hypertension treatment and control in the Genetic Epidemiology Network of Arteriopathy (GENOA) study. Am J Med 2004;116:676-681.

-17 Oliveira C, Pereira AC, de Andrade M, Soler JM, Krieger JE: Heritability of cardiovascular risk factors in a Brazilian population: Baependi heart study. BMC Med Genet 2008;9:32.

-18 Giolo SR, Soler JMP, Greenway SC, Almeida MAA, de Andrade M, Seidman JC, Seidman CE, Krieger JE, Pereira AC: Brazilian urban population genetic structure reveals a high degree of admixture. Eur J Hum Genet 2011; 19:111-116.

19 de Andrade M, Gueguen R, Visvikis S, Sass C, Siest G, Amos CI: Extension of variance components approach to incorporate temporal trends and longitudinal pedigree data analysis. Genet Epidemiol 2002;22:221-232. 
20 Lange K: Mathematical and Statistical Methods for Genetic Analysis, ed 2, rev. New York, Springer, 2002.

21 de Andrade M, Soler JMP: Multivariate polygenic mixed model in admixed population. Rev Estatistica UFOP 2014;3:200-209.

22 Bilodeau M, Duchesne P: Principal component analysis from the multivariate familial correlation matrix. J Multivar Anal 2002;82: 457-470.

23 Mardia KV, Bibby JM, Kent JT: Multivariate Analysis. London, Academic Press, 1979.

-24 Balding DJ, Nichols RA: A method for quantifying differentiation between populations at multi-allelic loci and its implications for investigating identity and paternity. Genetica 1995;96:3-12.
25 Purcell S, Neale B, Todd-Brown K, Thomas L, Ferreira MAR, Bender D, Maller J, Sklar P, de Bakker PIW, Daly MJ, Sham PC: PLINK: a toolset for whole-genome association and population-based linkage analysis. Am J Hum Genet 2007;81:559-575.

26 R Core Team: R: A Language and Environment for Statistical Computing. Vienna, R Foundation for Statistical Computing, 2014. ISBN 3-900051-07-0, URL http://www.Rproject.org/.

27 Chakraborty R, Weiss KM: Admixture as a tool for finding linked genes and detecting that difference from allelic association between loci. Proc Natl Acad Sci USA 1988;85: 9119-9123.
28 Goddard KA, Hopkins PJ, Hall JM, Witte JS: Linkage disequilibrium and allele frequency distributions for 114 single-nucleotide polymorphisms in five populations. Am J Hum Genet 2000;66:216-234.

29 Thomas DC, Witte JS: Point: Population stratification: a problem for case-control studies of candidate gene associations? Cancer Epidemiol Biomarkers Prev 2002;11:505512.

30 Cardon LR, Palmer LJ: Population stratification and spurious allelic association. Lancet 2003;361:598-604.

31 Marchini J, Cardon LR, Phillips MS, Donnelly P: The effects of human population structure on large association studies. Nat Genet 2004;36:512-517. 